\title{
PESQUISA E PÓS-GRADUAÇÃO NO BRASIL ATUAL: REFLEXÕES ENTRE O MODELO QUADRIENAL E O MULTIDIMENSIONAL
}

\author{
RESEARCH AND GRADUATE STUDIES IN BRAZIL CURRENT: REFLECTIONS \\ ON THE QUADRENNIAL AND MULTIDIMENSIONAL MODEL
}

\section{INVESTIGACIÓN Y ESTUDIOS DE POSGRADO EN BRASIL ACTUAL: REFLEXIONES SOBRE LA MODELO CUATRIENAL Y MULTIDIMENSIONAL}

\author{
Aníbal de Freitas Santos Júnior ${ }^{1}$ \\ José Antonio Gabriel Neto ${ }^{2}$
}

\begin{abstract}
Resumo
Este artigo tem como objetivo discutir, através de uma abordagem descritiva e exploratória, aliada à análise crítica e reflexiva, a estruturação e funcionamento do processo de avaliação dos Programas de Pós-graduação Stricto sensu (PPGSS), pela Coordenação de Aperfeiçoamento de Pessoal de Nível Superior (CAPES), no Brasil. São apresentados aspectos históricos, com ênfase na estruturação, desenvolvimento e avaliação, gerenciados pela CAPES. Parâmetros de alinhamento aos padrões internacionais, com dimensões e indicadores centrados em modelos de outros países, estão norteando a avaliação dos PPGSS no país e, nesse contexto, o modelo multidimensional seria uma solução? Questões alinhadas ao papel e função das Universidades e as pós-graduações brasileiras, a ausência de tratamento igualitário de financiamento às pesquisas pelo Governo (nas suas diversas esferas) e pelas Instituições, bem como a valorização das diferenças regionais são discutidas. Tais questões são heterogêneas e de difícil resolução, entretanto, busca-se aqui, uma reflexão sobre a promoção de uma Pós-graduação Stricto sensu com qualidade e de maior acesso á população, com vistas à qualificação humana.
\end{abstract}

Palavras-chave: Pós-graduação Stricto sensu; Avaliação; Indicadores; Modelos.

\begin{abstract}
This article aims to discuss, through a descriptive and exploratory approach, combined with critical and reflective analysis, the structuring and functioning of the evaluation process of the Stricto sensu Graduate Programs (PPGSS), by the Coordination for the Improvement of Level Personnel Superior (CAPES) in Brazil. Historical aspects are presented, with emphasis on structuring, development and evaluation, managed by CAPES. Alignment parameters to international standards, with dimensions and indicators centered on models from other countries, are guiding the evaluation of PPGSS in the country and, in this context, would the multidimensional model be a solution? Issues aligned with the role and function of Universities and Brazilian postgraduate courses, the absence of equal treatment of research funding by
\end{abstract}

\footnotetext{
1 Doutor em Química pela Universidade Federal da Bahia. Professor no Programa de Pós-graduação em Ciências Farmacêuticas e no Programa de Pós-graduação em Química Aplicada e no Programa de Pós-Graduação Multi-institucional e Multidisciplinar em Difusão do Conhecimento da Universidade do Estado da Bahia.

Contato: afjunior@uneb.br

ORCIDO: https://orcid.org/0000-0002-3022-0771

2 Doutor em Educação pela Universidade Federal do Ceará. Grupo de Pesquisas em Formação

Docente, História e Política Educacional.

Contato: prof.gabrielneto@outlook.com

ORCIDO: https://orcid.org/0000-0002-3136-6860
} 
the Government (in its various spheres) and by the Institutions, as well as the appreciation of regional differences are discussed. Such questions are heterogeneous and difficult to resolve, however, to discuss here, a reflection on the promotion of a Stricto sensu Graduate course with quality and greater access to the population, with a view to human qualification.

Keywords: Stricto sensu graduate studies; Evaluation; Indicators; Models.

\section{Resumen}

Este artículo tiene como objetivo discutir, a través de un enfoque descriptivo y exploratorio, combinado con un análisis crítico y reflexivo, la estructuración y funcionamiento del proceso de evaluación de los Programas de Posgrado Stricto sensu (PPGSS), por parte de la Coordinación de Perfeccionamiento del Personal de Nivel. Superior (CAPES) en Brasil. Se presentan aspectos históricos, con énfasis en estructuración, desarrollo y evaluación, gestionados por CAPES. Parámetros de alineación a estándares internacionales, con dimensiones e indicadores centrados en modelos de otros países, están orientando la evaluación de PPGSS en el país y, en este contexto, ¿el modelo multidimensional sería una solución? Se discuten temas alineados con el rol y función de las Universidades y los posgrados brasileños, la ausencia de un trato igualitario de la financiación de la investigación por parte del Gobierno (en sus diversas esferas) y de las Instituciones, así como la apreciación de las diferencias regionales. Este tipo de cuestiones son heterogéneas y difíciles de resolver, sin embargo, buscamos aquí una reflexión sobre la promoción de un curso de Postgrado Stricto sensu con calidad y mayor acceso a la población, con miras a la calificación humana.

Palabras clave: Estudios de postgrado Stricto sensu; Evaluación; Indicadores; Modelos.

\section{Introdução}

O Parecer n. 977/65, da Câmara de Ensino Superior (CES)/Conselho Federal de Educação (CFE), relatado pelo professor Newton Sucupira, forneceu a base conceitual da Pós-graduação Stricto sensu (PGSS) no país, estabelecendo os mestrados e doutorados acadêmicos. Por conseguinte, com a criação da Coordenação de Aperfeiçoamento de Pessoal de Nível Superior (CAPES), o Sistema Nacional de Pós-graduação (SNPG) tem se aperfeiçoado no que se refere à avaliação dos Programas de Pós-graduação Stricto sensu (PPGSS), visando a formação de recursos humanos qualificados para o mercado acadêmico e não-acadêmico, bem como o fortalecimento das bases científica, tecnológica e de inovação do país, conforme o Plano Nacional de Pós-Graduação (PNPG) - 2011/2020 (BRASIL, 2010). Logo, a avaliação do Sistema Nacional de Pós-Graduação, estabelecida a partir de 1998, é atividade essencial para assegurar e manter a qualidade dos cursos da PGSS brasileira. 
O SNPG registra, atualmente, um pouco mais de 4.600 PPGSS e, portanto, é importante compreender como funciona a pós-graduação brasileira, com base em um resgate de parte de sua memória. Através de uma abordagem descritiva e exploratória, aliada à análise crítica e reflexiva, este texto discute como está estruturada e como funciona o processo de avaliação dos PPGSS, pela CAPES e, as perspectivas e mudanças a serem implementadas, a partir do ano de 2021, com a introdução da avaliação multidimensional, já em discussão há algum tempo no âmbito do Ministério da Educação (MEC) e na comunidade acadêmica.

\section{Histórico e evolução da pós-graduação no Brasil}

Para entender como a pós-graduação no Brasil teve impulso, é importante voltar um pouco no tempo e analisar as políticas científicas das décadas anteriores àquela que se considera como marco inicial, a de 1960. As primeiras instituições de ensino superior do país foram sendo formadas a partir, principalmente, da chegada da Família Real ao país, em 1808. Contudo, eram escolas separadas e sem um constituinte efetivo de Universidade, o que só aconteceria no século XX. Dessa forma, a partir da década de 1930, se inicia a oferta de cursos de especialização Lato sensu em território nacional. Hoje, os cursos chamados de especializações são cursos regulamentados pela resolução número 1, de 6 de abril de 2018 (BRASIL, 2018). Além disso, não conferem diploma, mas certificado de conclusão.

Os cursos de especialização tiveram início ainda na década de 20 do século passado, ou seja, já é uma trajetória longa e considerável. Em 1931, a partir do decreto 19.851, ficou estabelecido que as Universidades brasileiras poderiam conferir o título de doutor a candidatos que concluíssem cursos normais, técnicos ou científicos e apresentassem contribuição inédita ao seu respectivo campo do saber através de apresentação de tese (BRASIL, 1931). Esse título estava destinado àqueles que tinham por vontade seguir a carreira docente.

No entanto, Cury (2005), assim como CAPES (2011) entenderam que o primeiro curso do tipo no território nacional foi o doutorado em Direito, da Universidade Federal de Minas Gerais, criado em 1931. Até a criação da própria CAPES, 20 anos depois, era o único curso do tipo em todo o território nacional. Há de se 
distinguir, porém, curso de pesquisa, uma vez que um curso demanda organização institucional, curricular e docente, enquanto que a segunda não necessariamente segue essa ordem. Pode-se considerar, dessa forma, que o título de doutor era feito de forma pouco articulada e de uma maneira quase que artesanal. Há de se considerar, ainda, que não havia o curso de mestrado, hoje uma etapa fundamental para os aspirantes à obtenção do grau de doutorado, sendo que o título de doutor era obtido diretamente.

Portanto, não havia uma institucionalidade da pós-graduação ou órgão que o regulasse como há atualmente. A pesquisa, então, não obedecia aos critérios de estar ligada a um programa específico sendo então determinada uma direção curricular. $\mathrm{E}$, além disso não havia um orientador, mas sim um diretor de pesquisa, apesar dos dois serem hoje equivalentes. Nota-se, por conseguinte, que a história da pós-graduação no Brasil tem, portanto, uma trajetória histórica de, pelo menos, 90 anos.

Os cursos daquela época, é claro, eram muito diferentes dos atuais. Em primeiro lugar, não havia uma normativa que controlasse o formato e a oferta desses cursos como há agora. Adicionalmente, especializar-se em termos Lato sensu era praticamente uma coisa inexistente no Brasil, até pelo menos os anos 50 . Mestrado e doutorado no país nem existiam em conjunto, pelo menos, até o final dessa mesma década.

Cabe dizer, porém, que a partir da II Guerra Mundial esse cenário se modifica. Havendo a necessidade de uma modernização por conta da modificação da geopolítica internacional, o Brasil também entrou em um novo patamar em termos de pesquisa. Em fins da década de 1950, houve a criação dos primeiros cursos de mestrado e doutorado no Brasil. O primeiro mestrado do Brasil foi o curso de Hortaliças pertencente à Universidade Federal de Viçosa, em Minas Gerais, hoje abrigando os cursos de mestrado e doutorado em Fitotecnia (Produção Vegetal).

Em 1964 há uma modificação no contexto político e educacional no Brasil. O golpe militar transforma os setores do estado e também os entes privados. $O$ golpe foi sendo germinado, aos poucos, desde a morte de Getúlio Vargas para impedir um suposto golpe esquerdista, o que nunca aconteceu. Dessa forma, a pós-graduação passou a ser um dos eixos de debate dentro do MEC, era uma demanda que fez parte das discussões no âmbito da CAPES na década anterior. No contexto da 
ditadura militar, a CAPES também iniciou o fomento aos estudantes de pósgraduação, através da concessão de bolsas de estudos para aqueles que desejavam cursar mestrado e doutorado. $O$ decreto $n^{\circ} .54 .356 / 64$, assinado pelo então presidente Castelo Branco, concedia bolsas a brasileiros no país e no exterior bem como a estrangeiros que desejassem realizar estudos de pós-graduação em solo nacional.

Assim, entre os anos de 1961 e 1974, ocorreu o que pode ser chamada de primeira grande expansão da pós-graduação brasileira. Essa primeira expansão marcou o nascimento e o início da consolidação da pós-graduação como política de estado. Apesar de institucionalizada em 1965, apenas com a reforma de 68 é que houve a verdadeira regulamentação do ensino de pós-graduação no Brasil. Em seu artigo 17, a reforma regulamentou quem podem ser os ingressantes em cursos de pós-graduação, ou seja, portadores de diplomas de graduação (BRASIL, 1968).

No período de vigência do primeiro PNPG foi criada a tão conhecida avaliação de cursos de pós-graduação da Capes. Criado em 1977, foram instituídas as comissões de assessores por área, e foi estabelecido o Conselho Técnico-Científico da Educação Superior (CTC-ES), sistema que vigora até os dias atuais (BARATA, 2015). A avaliação era feita a partir de conceitos, que variavam entre a letra $A$ e a letra E. Esses conceitos, como acontece hoje com o sistema numérico, tinham por objetivo dar uma ideia da qualidade de cada programa de pós-graduação a partir de critérios objetivos.

A avaliação da CAPES teve início durante a presidência do professor gaúcho Darcy Closs, que presidiu a Instituição entre 1974 e 1979. Naquele tempo, a expansão da pós-graduação, pesquisa e desenvolvimento era uma questão muito importante para o estado brasileiro, sob orientação da Agência dos Estados Unidos para o Desenvolvimento Internacional (do inglês, United States Agency for International Development - USAID), órgão dos Estados Unidos da América para o desenvolvimento internacional.

Havia a ideia de que a avaliação poderia ser um termômetro para medir qualitativamente a pós-graduação brasileira, uma vez que o aumento entre o início da década de 1960 e fins dos anos 70 havia sido exponencial. Dessa forma, entendia-se que era necessário um controle maior para consolidar a qualidade da pós-graduação em território nacional. A primeira avaliação foi efetivamente constituída em 
1978, ainda na gestão Closs. Entretanto, foi Cláudio de Moura Castro, presidente da CAPES entre 1979 e 1982, que houve uma introdução desta como algo permanente e cotidiana no trabalho dos cientistas nacionais. A CAPES então avaliava cada programa através de um sistema em formas de letras que possuía o nome de "conceito" A (muito bom), B (bom), C (regular), D (fraco) e E (insuficiente) (FERREIRA; MOREIRA, 2002). A avaliação, não estando acessível ao público, era disponibilizada apenas às instituições que possuíam cursos de mestrado e doutorado em funcionamento.

Esse perfil de avaliação revelou que a ideia sempre foi quantificar a qualidade dos PPGSS com critérios objetivos, que pudessem ser medidos em números para estabelecer quais instituições tem programas com maior prestígio, sendo que esse sistema ainda vigorou pelo menos até a avaliação do ano de 2017. Com isso, é possível aferir, portanto, que as noções de centro e periferia já estavam presentes na pós-graduação brasileira desde os seus primórdios, sendo que os grandes centros, geograficamente localizados em centros urbanos maiores e com maior dinamicidade econômica, sempre tiveram, no geral, mais programas com notas mais altas e, por conseguinte, mais recursos para o seu desenvolvimento.

Em 1981, o Conselho Nacional de Pós-graduação foi extinto, sendo que a CAPES passou, a desempenhar papel totalmente central em todos os âmbitos: fomento, acompanhamento, coordenação, avaliação e proposição de políticas para o setor científico nacional, juntamente ao Conselho Nacional de Desenvolvimento Científico e Tecnológico (CNPq).

Foi durante a presidência da professora Eunice Durham frente ao órgão do MEC, no governo Collor que houve a mudança na avaliação para os moldes atuais. Embora um pouco diferente, aquele seria o modelo para a avaliação tal como se conhece agora. Existia o entendimento, naquela época, de que a avaliação precisava de mudanças. A maioria dos cursos conseguia conceitos A e B, cerca de $90 \%$ (FERREIRA; MOREIRA, 2002). As notas 1 a 5 substituíram os conceitos anteriores, passando então a adoção de um sistema numérico. Em 1998, porém, entra em vigor o modelo atual que será explicado, logo a seguir. No início, eram realizadas avaliações trienais, ou seja, a cada 3 (três) anos. A partir de 2013, porém, elas passam a ser quadrienais, a cada 4 anos. 


\section{Mas como funciona a avaliação?}

Para responder a essa pergunta, em primeiro lugar é preciso destacar como a CAPES está organizada, em um total de 49 (quarenta e nove) áreas, que estão inseridas nas grandes Áreas e nos Colegiados, como demonstrado na Figura 1.

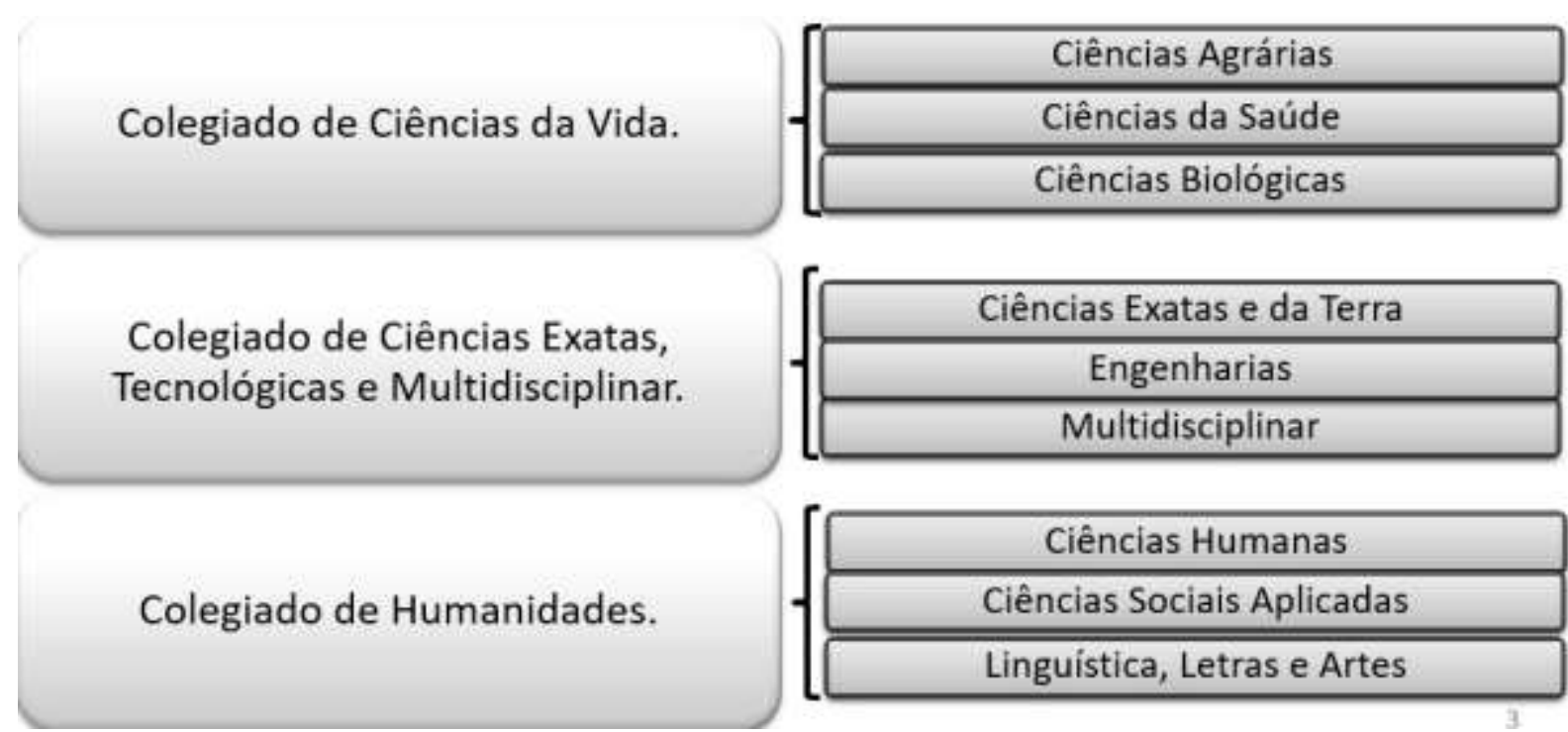

Figura 1. Colegiado e áreas de conhecimento da CAPES. (Fonte: BRASIL, 2018).

Cada Colégio é responsável por três grandes Áreas do conhecimento, sendo que cada grande área tem seus próprios campos.

O Colegiado de Ciências da Vida conta com:

$>$ Ciências Agrárias - Agronomia, Medicina Veterinária, Engenharia Florestal etc.

$>$ Ciências da Saúde - Medicina, Enfermagem, Fisioterapia, Terapia Ocupacional, Fonoaudiologia, entre outros

$>$ Ciências Biológicas - Bioquímica, Botânica, Ecologia, Fisiologia etc.

O Colegiado de Ciências Exatas, Tecnológicas e Multidisciplinar:

$>$ Ciências Exatas e da Terra: Física, Matemática, Geociências, Química, Astronomia, entre outros.

$>$ Engenharias: Engenharias Civil, Mecânica, Elétrica, de Produção, Metalúrgica etc.

$>$ Biotecnologia, Ensino, Materiais, Ciências Ambientais.

Por fim, o Colegiado de Humanidades 
> Ciências Humanas: História, Educação, Filosofia, Geografia, Sociologia, entre outros.

> Ciências Sociais Aplicadas: Turismo, Direito, Economia, Administração, Contabilidade etc.

> Linguística, Letras e Artes: Linguística, Literatura, Artes (Dança, Música, Artes Cênicas, Artes Plásticas, entre outros).

Cada Área tem uma Comissão de avaliação própria, com coordenadores (geral, adjunto e coordenador de programas do tipo profissionais) e consultores, que se reúne, em Brasília, no ano da avaliação, atribuindo uma nota para cada PPGSS, de acordo com determinados critérios. Embora possam haver sutis variações, as grandes categorias para avaliação dos PPGSS, são as mesmas para todas as Áreas de conhecimento.

Iniciar, prospectar, gerir e avaliar um curso de Pós-graduação Stricto sensu (mestrado e/ou doutorado) no país é uma tarefa que requer muita dedicação, responsabilidade e acompanhamento dos processos e normativas da CAPES! O processo de avaliação de Programas de Pós-graduação Stricto sensu, no Brasil, é realizado em dois momentos: i) inicialmente, na submissão/apresentação de Propostas de cursos Novos (APCN) e, ii) na avaliação periódica, com periodicidade quadrienal. Partindo-se desse cenário, os relatórios das áreas, fichas de avaliações e demais documentos disponíveis no sítio eletrônico da CAPES devem ser consultados, sendo essenciais para melhor compreensão de ambos as etapas supracitadas.

Os processos avaliativos são operacionalizados pelas coordenações de cada área de conhecimento (atualmente, são 49 áreas), que atribuem notas/conceitos aos PPGSS em uma escala de 1 a 7 , reservando-se as notas 6 e 7 para os cursos de excelência. Os indicadores destas avaliações são propostos pela CAPES, em consonância com a Política Nacional de Pós-Graduação do país. É salutar que tais indicadores sejam adequados e reavaliados constantemente; afinal, os cenários políticos, socioeconômicos e culturais são variados, conforme os diastrofismos locais e regionais (HORTALE, 2003; MIORANZA; AQUINO, 2009). Entretanto, parâmetros lineares e gerais devem ser instituídos para adequação dos PPGSS aos indicadores avaliativos propostos. De acordo com a CAPES, a avaliação dos PPGSS, principalmente os cursos de excelência, visa que os mesmos alcançam desempenho equivalente aos centros de referência internacional. Neste cenário, a premissa é de 
que "a nota do programa esteja atrelada ao grau de maturidade que o programa atingiu dentro do sistema”. E, para tanto, a avaliação dos PPGSS pauta-se pelo mesmo conjunto de quesitos e itens avaliativos no intuito de promover a equivalência de desempenho entre programas com as mesmas notas em diferentes áreas de avaliação (BRASIL, 2019a).

O instrumento avaliativo, denominado "Ficha de avaliação" é estabelecido por cada área do Conhecimento, com a finalidade de padronização dos quesitos e itens. A métrica e o peso atribuído a cada quesito/item variam conforme a área de avaliação. No quadriênio anterior, que compreendeu ao período de 2013 a 2016, a Ficha de Avaliação englobou os quesitos (Proposta do Programa, Corpo Docente, Corpo Discente, Teses e Dissertações, Produção Intelectual, e Inserção Social). Para cada item, foram atribuídos os conceitos: "Muito Bom", "Bom", "Regular", "Fraco" e "Insuficiente" (BRASIL, 2019a). Por conseguinte, trata-se de um processo meritocrático, criterial e normativo que utiliza padrões pré-estabelecidos para todos os PPGSS, que comparados ao "desempenho médio" dos demais programas daquela área de avaliação.

A partir do exposto até aqui, observa-se que os PPGSS são estimulados a atingirem padrões cada vez "maiores", estimulando uma competição entre os mesmos. Isso é benéfico ou maléfico? Depende....e muito de cada PPGSS, da sua inserção e acolhimento institucional, lócus, investimento, dentre outros fatores. Certamente, este processo de avaliação "força" e impõe uma dinâmica de alteração e elevação de padrões de qualidade, uma vez que, caso não obtenham uma avaliação satisfatória, no mínimo 'Regular”, o Programa pode ser descredenciado pela CAPES, que traduz um processo, também, regulatório por este órgão.

No cenário atual, para a avaliação do quadriênio 2017-2020, a CAPES propõe um modelo avaliativo com 3 parâmetros: 1) Programa; 2) Formação e, 3) Impacto na sociedade. Para a CAPES, a proposta avaliativa consiste na redução do número de quesitos, destacando aqueles que "verdadeiramente" discriminam a qualidade dos programas, dando ênfase a formação discente, com ênfase maior à avaliação de resultados do que de processos, valorizando a construção de indicadores. O primeiro quesito "Programa" consiste em avaliar o funcionamento, estrutura e planejamento do programa de pós-graduação em relação ao seu perfil e seus objetivos; o 
segundo, "Formação" tem seu foco na qualidade dos recursos humanos formados, levando em conta a atuação dos docentes e a produção de conhecimento diretamente associada às atividades de pesquisa e de formação do programa e, o terceiro "Impacto na sociedade", avaliar os impactos gerados pela formação de recursos humanos e a produção de conhecimentos do PPGSS (BRASIL, 2019b). Deste panorama atual, destacam-se os processos, procedimentos e resultados da autoavaliação do programa, com foco na formação discente e produção intelectual, que deve estar atrelada ao Plano de Desenvolvimento Institucional (PDI), às Pró-reitorias e demais setores internos da Universidade, bem como a parcerias intra e extra institucionais.

Para o quadriênio 2021 - 2024, a CAPES vem reformulando os seus critérios avaliativos e, portanto, se baseando em indicadores e plataformas de classificação e avaliação - como, por exemplo, o U-Multirank e os indicadores bibliométricos, tais como o sistema de categorização de periódicos da Clarivate Analytics e da Web of Science (WoS), que buscam comparar o desempenho das instituições a partir de uma abordagem multidimensional. O U-Multirank (instutuída pela Comissão Européia de avaliação) aborda as seguintes dimensões distintas, dentre estas: (1) ensino e aprendizagem, (2) pesquisa, (3) transferência de conhecimento, (4) orientação internacional e (5) engajamento regional, onde nenhuma há hierarquia entre as dimensões. Portanto, esta proposta avaliativa busca aproximar a avaliação dos PPGSS aos padrões avaliativos internacionais (BRASIL, 2019a; U-MULTIRANK, 2019). Há de se questionar e refletir sobre o uso destes indicadores e plataformas, uma vez que há diferenças substanciais entre estas ferramentas e a proposta de avaliação da CAPES, pois a priori, todas as dimensões possuem a mesma importância. E, a CAPES visa acreditar a qualidade dos programas de pós-graduação por meio da comparação de desempenho de PPGSS, a partir de critérios definidos pela comunidade acadêmica.

Neste cenário, a CAPES almeja um processo avaliativo inovador, com a atribuição de diferentes notas a cada uma das dimensões de desempenho:

“...Cada PPG, e Instituição por consequência, teria um desempenho (conceito) para cada eixo (ou dimensão) da avaliação, permitindo aflorar desempenhos diferentes para cada dimensão, dando espaço para reconhecer a diversidade e a qualidade dos PPGs e Instituições em cada eixo (dimensão) da avaliação" (BRASIL, 2018). 
Com isso, para a avaliação quadrienal de 2017-2020, a CAPES propõe um modelo multidimensional com 5 dimensões para avaliação: 1) Formação de Pessoal; 2) Pesquisa; 3) Inovação e Transferência de Conhecimento; 4) Impacto na Sociedade; 5) Internacionalização. Com isso, é notório que os PPGSS acompanhem as mudanças propostas e se (re)adequem ao sistema avaliativo proposto e, nesta perspectiva desafios serão necessários e essenciais com a finalidade de fortalecimento e consolidação. Nesta lógica, aspectos micro (internos) e macro (externos) serão focos de discussões entre os PPGSS, alinhados às propostas e políticas de pesquisa e pósgraduação institucionais, bem como àquelas propostas pelo Ministério da Educação, a partir da CAPES.

O quesito "Formação de Pessoal" foca na atratividade do Curso/Programa, valorizando a formação e satisfação de mestres e doutores; a diversidade de oferta de oportunidades de formação, valorizando o envolvimento de orientandos, orientadores e pesquisadores externos em atividades formativas; e, um dos pontos de sucesso desse indicador reflete, além da formação, a inserção profissional e o desempenho de egressos no mercado de trabalho. Percebe-se aqui, que se ratifica o foco da qualidade da formação e qualificação dos egressos e, ademais, se destaca a inserção destes no mercado de trabalho, como forma de medida da qualidade do PPGSS. A introdução de novas ferramentas metodológicas (ativas e colaborativas) que valorizem as competências e habilidades, também, fortalece este quesito de avaliação.

O quesito "Pesquisa" ratifica a importância da produção técnica e científica através da regularidade e participação de docentes e discentes nos produtos do PPGSS. Ainda, há (e se deve ter, na perspectiva dos autores deste texto) a preocupação do impacto (local, regional, nacional e, principalmente internacional) destas produções, associadas à difusão do conhecimento através de redes colaborativas de pesquisas, com parcerias nacionais e internacionais, inclusive, com captação de recursos em órgãos de fomento. Atrelado a isso, destaca-se o quesito "Inovação e Transferência de Conhecimento" com a perspectiva de inovações sociais e culturais relevantes, através de projetos que contribuam com a sustentabilidade ambiental e tecnológica, através de parcerias com empresas, centros de pesquisas e 
outros setores da economia, com foco na possibilidade de transferência de tecnologias e do conhecimento, em geral.

O quesito "Impacto na Sociedade" é também, de extrema relevância para a contextualização do PPGSS que, deve focar no desenvolvimento econômico e social, regional e nacional. Os principais itens avaliados neste quesito estão voltados aos impactos e caráter inovador da produção intelectual (bibliográfica, técnica e/ou artística) em função da natureza do programa; ao destino, atuação e avaliação dos egressos do programa em relação à formação recebida; bem como, aos impactos da inserção social, econômica, visibilidade e internacionalização do programa. Este quesito possui relação com o último quesito proposto, a "Internacionalização" pois se relaciona com aspectos relacionados às redes colaborativas e ao financiamento de pesquisas internacionais, favorecendo intercâmbios entre discentes e docentes em atividades internacionais, ampliando-se as experiências de vida e na expertise para solucionar problemas. Com isso, aumenta-se a visibilidade do PPGSS e se promove o fortalecimento e a consolidação.

\section{Considerações Finais}

Algumas reflexões e discussões acerca do processo avaliativo da PGSS no país vêm acontecendo ao longo do tempo. Qual seria a melhor forma de avaliar a pósgraduação brasileira, com diversos cenários e perspectivas das regiões do país, bem como maior alinhamento aos padrões internacionais? O modelo multidimensional seria uma solução? As dimensões e indicadores propostos estão centrados no modelo europeu de ensino superior e, estariam alinhados ao papel e função das universidades e as pós-graduações brasileiras? A ausência de tratamento igualitário de financiamento às pesquisas pelo Governo (nas suas diversas esferas) e pelas Instituições, bem como as diferenças regionais seriam valorizadas na avaliação? São perguntas, com variáveis heterogêneas e de difícil resolução, entretanto, segue-se a luta para promover uma PGSS de qualidade e de maior acesso á população, com vistas à qualificação humana.

\section{Referências}


BARATA, R. B. A ABRASCO e a pós-graduação stricto sensu em Saúde Coletiva. In: LIMA, N. T.; SANTANA, J. P.; PAIVA, C. H. A. (Eds.) Saúde Coletiva: a ABRASCO em 35 anos de história. Rio de Janeiro: Fiocruz/Abrasco, 2015.

BRASIL. Decreto-lei n. 19.851 de 11 de abril de 1931. Estatuto das universidades brasileiras. Brasília, 1931. Disponível em: http://www.camara.gov.br/internet/ InfDoc/novoconteudo/legislacao/republica/Leis1931vl625p/pdf37.pdf. Acesso em: 15 ago. 2020.

BRASIL. Lei no 5.540, de 28 de novembro de 1968. Disponível em: http://www2.camara.leg.br/legin/fed/lei/1960-1969/lei-5540-28-novembro-1968359201-publicacaooriginal-1-pl.html Acesso em: 10 nov. 2020.

BRASIL. Ministério da Educação. Coordenação de Aperfeiçoamento de Pessoal de Nível Superior (CAPES). Relatório Técnico DAV - Avaliação Multidimensional de Programas de Pós-Graduação. Brasília, 2019a. Disponível em: https://www.gov.br/capes/pt-br/centrais-de-conteudo/23072020-dav-multi-pdf. Acesso em: 20 out 2020.

BRASIL. Ministério da Educação. Coordenação de Aperfeiçoamento de Pessoal de Nível Superior (CAPES). Ficha de Avaliação - Grupo de Trabalho. Brasília, 2019b. Disponível em: http://www.prppg.ufpr.br/site/wpcontent/uploads/2020/07/10062019 fichaavaliao.pdf. Acesso em: 20 out 2020.

BRASIL. Ministério da Educação. Coordenação de Aperfeiçoamento de Pessoal de Nível Superior (CAPES). Aprimoramento do Modelo de Avaliação da PG. Documento Final da Comissão Nacional de Acompanhamento do PNPG 20112020. Brasilia, 2018. Disponível em: https://www.gov.br/capes/pt-br/centrais-deconteudo/2018-pnpg-cs-avaliacao-final-10-10-18-cs-final-17-55-pdf. Acesso em: 20 out 2020.

BRASIL. Ministério da Educação. Coordenação de Aperfeiçoamento de Pessoal de Nível Superior (CAPES). Plano nacional de pós-graduação [PNPG] 2011-2020. v. 1 e 2. Brasília: CAPES, 2010. Disponível em: https://www.gov.br/capes/ptbr/centrais-de-conteudo/livros-pnpg-volume-i-mont-pdf. Acesso em: 20 dez 2020.

CURY, C. R. J. Os Fora de série na escola. Campinas: Autores Associados, 2005.

FERREIRA, M. M.; MOREIRA, R. L. Capes. 50 anos. Depoimento ao CPDOC/ FGV. Brasília, 2002. Disponível em: http://www.dominiopublico.gov.br/ download/texto/me001600.pdf. Acesso em: 5 out. 2020.

HORTALE, V. A. Modelo de avaliação CAPES: desejável e necessário, porém, incompleto. Cad. Saúde Pública, v. 19, n. 6, p. 1837-1840, 2003.

MIORANZA, C.; AQUINO, A. F. Modelo Multidimensional de Avaliação da Qualidade Educacional - MULTQUALED. Rev. Iberoam. Educ. , v. 49, n. 6, p. 1-20, 2009. 
U-MULTIRANK. U-Multirank Project. 2019. Disponível em: http://twixar.me/5d0n/. Acesso em: 20 out 2020.

Manuscrito recebido em: 27 de outubro de 2020

Aprovado em: 30 de dezembro de 2020

Publicado em: 31 de dezembro de 2020 